ARTICLE

Received 3 Oct 2013 | Accepted 13 Dec 2013 | Published 16 Jan $2014 \quad$ DOl: 10.1038/ncomms4104

\title{
Split-ball resonator as a three-dimensional analogue of planar split-rings
}

\author{
Arseniy I. Kuznetsov ${ }^{1}$, Andrey E. Miroshnichenko ${ }^{2}$, Yuan Hsing Fu', Vignesh Viswanathan ${ }^{3}$, Mohsen Rahmani ${ }^{1}$, \\ Vytautas Valuckas ${ }^{1,3}$, Zhen Ying Pan ${ }^{1}$, Yuri Kivshar ${ }^{2}$, Daniel S. Pickard ${ }^{3}$ \& Boris Luk'yanchuk ${ }^{1}$
}

Split-ring resonators are basic elements of metamaterials, which can induce a magnetic response in metallic nanosctructures. Tunability of such response up to the visible frequency range is still a challenge. Here we introduce the concept of the split-ball resonator and demonstrate the strong magnetic response in the visible for both gold and silver spherical plasmonic nanoparticles with nanometre scale cuts. We realize this concept experimentally by employing the laser-induced transfer method to produce near-perfect metallic spheres and helium ion beam milling to make cuts with the clean straight sidewalls and nanometre resolution. The magnetic resonance is observed at $600 \mathrm{~nm}$ in gold and at $565 \mathrm{~nm}$ in silver nanoparticles. This method can be applied to the structuring of arbitrary three-dimensional features on the surface of nanoscale resonators. It provides new ways for engineering hybrid resonant modes and ultra-high near-field enhancement.

\footnotetext{
${ }^{1}$ Data Storage Institute, A*STAR (Agency for Science, Technology and Research), 5 Engineering Drive 1, 117608 Singapore, Singapore. ${ }^{2}$ Nonlinear Physics Centre, Research School of Physics and Engineering, Australian National University, Canberra, Australian Capital Territory 0200, Australia. ${ }^{3}$ Department of Electrical and Computer Engineering, National University of Singapore, 4 Engineering Drive 3, 117583 Singapore, Singapore. Correspondence and requests for materials should be addressed to A.I.K. (email: arseniy_k@dsi.a-star.edu.sg).
} 
O ne of the main challenges in the field of plasmonics and metamaterials during the last decade is to engineer nanostructures with strong magnetic and electric dipole resonances at optical frequencies ${ }^{1-5}$. Getting these two resonances together in the same frequency range can lead to unique material properties associated with near-zero or even negative effective refractive index. The concept of the split-ring resonator (SRR), which provides a strong magnetic dipole response of metallic structures, was theoretically introduced by Pendry et al. ${ }^{6}$ in 1999. Since then, many efforts to experimentally demonstrate a magnetic resonance of metallic structures have been reported, first in the gigahertz range ${ }^{7}$, then in the terahertz range $^{8}$, and finally at optical frequencies ${ }^{9}, 10$. It has been shown that scaling down the size of the SRR linearly increases the magnetic resonance frequency. However, this linear dependence saturates close to the visible spectral range mainly due to non-ideality of plasmonic metals at these frequencies ${ }^{10,11}$. Further control of the resonance position is possible using optimization of the design of SRRs ${ }^{12,13}$. More advanced designs, such as cut-wire pairs ${ }^{4,15}$, fish-net structures ${ }^{16,17}$, and nanoparticle clusters ${ }^{18,19}$, have also been implemented to facilitate metamaterial fabrication and further increase their magnetic resonance frequency. However, until now there have been few experimental demonstrations of resonant magnetic response of metallic nanostructures in the visible spectrum ${ }^{18-26}$. Strong dissipative losses of plasmonic nanostructures in the visible spectral range significantly limit the possibilities of shifting the magnetic resonance to higher frequencies. These losses arise due to imperfections of the nanostructures at the sub- $100 \mathrm{~nm}$ scale and intrinsic losses inherent to plasmonic metals in this frequency range.

One of the peculiarities of the magnetic response of metallic nanostructures is its possible overlap with a strong electric resonance provided by the same metallic elements. For example, it can be analytically demonstrated in the quasi-static limit that the magnetic dipole resonance of SRRs is always accompanied by the electric dipole resonance generated inside the gap of SRRs, being of the same or even higher order of magnitude ${ }^{27,28}$. Such combined electric and magnetic response of SRRs is appropriate to call an $\mathrm{LC}$ resonance to account for different multipolar modes contributing to the resonance peak.

The use of low-loss resonant high-refractive index dielectric nanoparticles having strong Mie resonances of both electric and magnetic origins ${ }^{29-35}$ can partially solve this problem and shift the magnetic resonance frequency to the blue spectral range $29,31,34$. In this case, a pure magnetic dipole resonance with very minor influence of other multipolar modes can be generated.

In this Article, we introduce and experimentally demonstrate a new concept of a strong magnetic dipole resonance tuneable almost throughout the whole visible spectral range using standard plasmonic metals such as gold and silver. The key aspect is a nanometre-size cut fabricated inside an almost perfectly spherical plasmonic nanoparticle. Such a threedimensional (3D) spherical design allows for shifting of the LC-type magnetic resonance down to the main electric dipole resonance wavelength in the visible spectral range. Partial contribution of different multipolar modes into the resonant scattering is analysed and strong magnetic dipole contribution is demonstrated. Experimentally, this novel design is realized by combining the almost perfect spherical nanoparticles achievable with the laser-induced transfer method (LIT) with the unprecedented patterning fidelity at nanometre scale dimensions characteristic of milling with a focused helium ion beam. Towards the understanding of the physics of such nanostructures, nearfield enhancement and Poynting vector flow around them are also discussed.

\section{Results}

Numerical simulations. A schematic representation of a metallic sphere with a cut, split-ball resonator (SBR), is shown in Fig. 1. The nanocut is introduced on top of the sphere making it similar to $3 \mathrm{D}$ upright SRRs $\mathrm{s}^{36,37}$. In this case, the coupling of light to the magnetic resonance mode should be more efficient due to both electric and magnetic field contributions.

To analyse the ability of the SBR to support the magnetic dipole resonance, we performed numerical simulations of its optical spectral properties using commercial CST Microwave Studio software. Results are shown in Fig. 2 for a gold sphere with diameter of $200 \mathrm{~nm}$ having a $50 \mathrm{~nm}$ wide cut going down to the centre of the sphere. The nanostructure is located in free space and excited by a plane wave directed from the top (antiparallel to $z$ axis) with two different polarizations, parallel (along $y$ axis) and perpendicular (along $x$ axis) to the nanocut direction. Total scattering and absorption efficiencies by the nanostructure are shown in Fig. 2a (see Methods for details).

When the incident light polarization is parallel to the cut, only a single electric dipole resonance is excited inside the SBR, similar to a spherical nanoparticle without a cut (Fig. 2a). The electric dipole nature of this resonant peak is confirmed by electric and magnetic field distribution at the resonance wavelength (Fig. $2 b, c$ ) and decomposition of the near-field into spherical multipoles (Fig. 2g, see detailed discussions below). When incident light polarization is perpendicular to the cut, two resonant peaks appear in the scattering spectrum: the first weaker peak is close to the position of the electric resonance, and the second, much stronger peak is at the longer wavelengths (see Fig. 2a). This second resonant peak corresponds to the so-called LC resonance excited in the SBR similar to the case of $\mathrm{SRRs}^{6-13,28}$. The scattering intensity at this resonance is defined by a combination of strong electric and magnetic dipole contributions (Fig. 2f, see detailed discussions below). The electric field at this resonant wavelength is strongly enhanced inside the cut generating circulating electric currents flowing around the nanocut inside the nanoparticle (Fig. 2d). On the other hand, the magnetic field

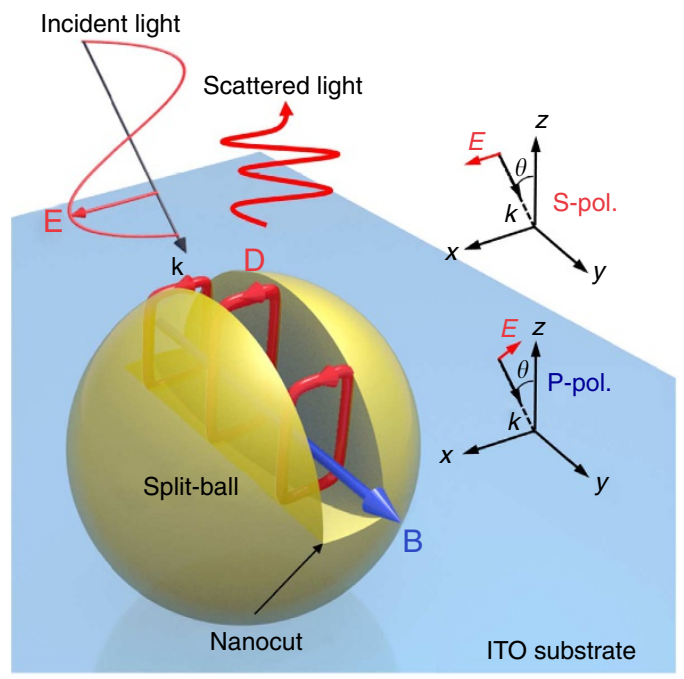

Figure 1 | Schematic representation of the SBR and geometry of spectral measurements. The nanocut is introduced on top of the metallic sphere. The incident light is directed onto the SBR from one side facing the nanocut at an angle of $58.5^{\circ}$ to the surface normal. Two incident light polarizations are studied: $s$ (perpendicular) and $p$ (parallel) to the nanocut plane. The scattered light is collected from top by a microscope objective lens with $0.55 \mathrm{NA}$. 

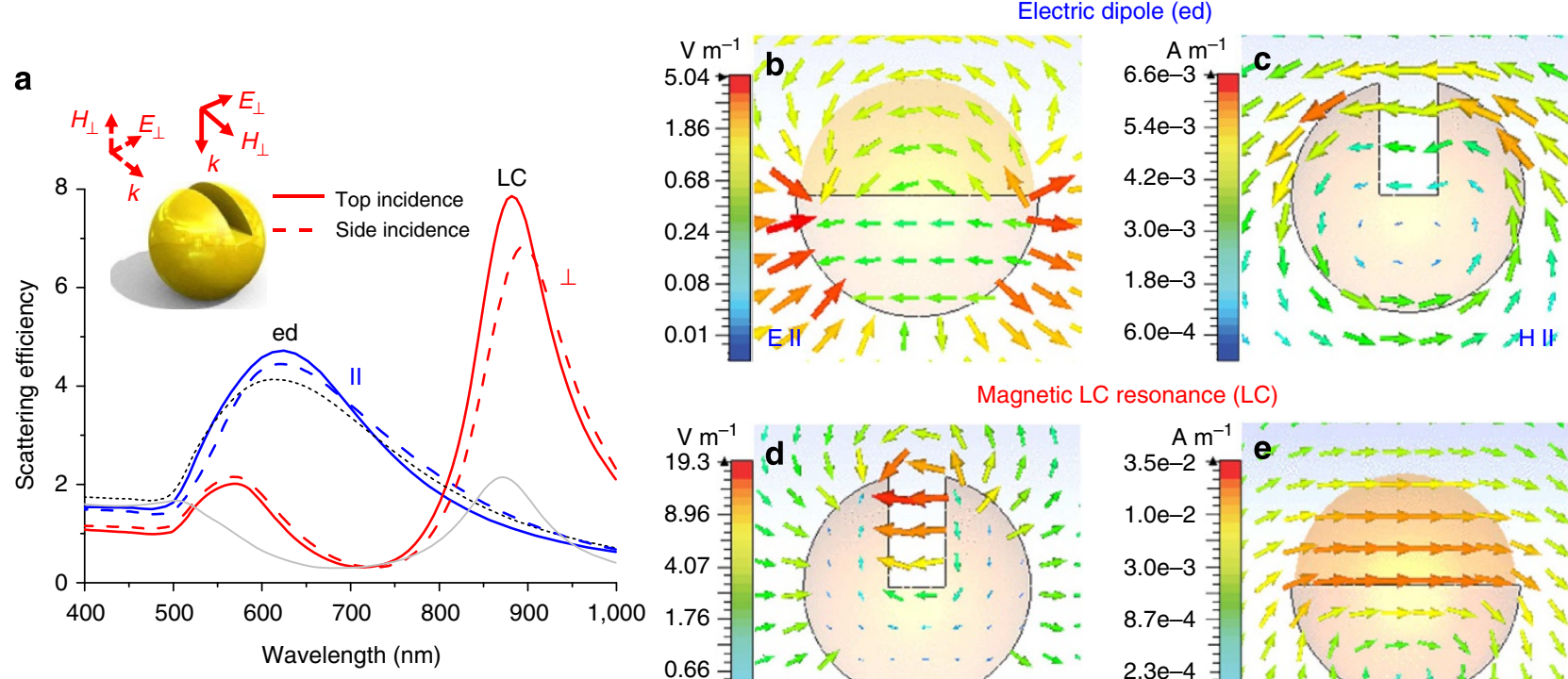

Magnetic LC resonance (LC)
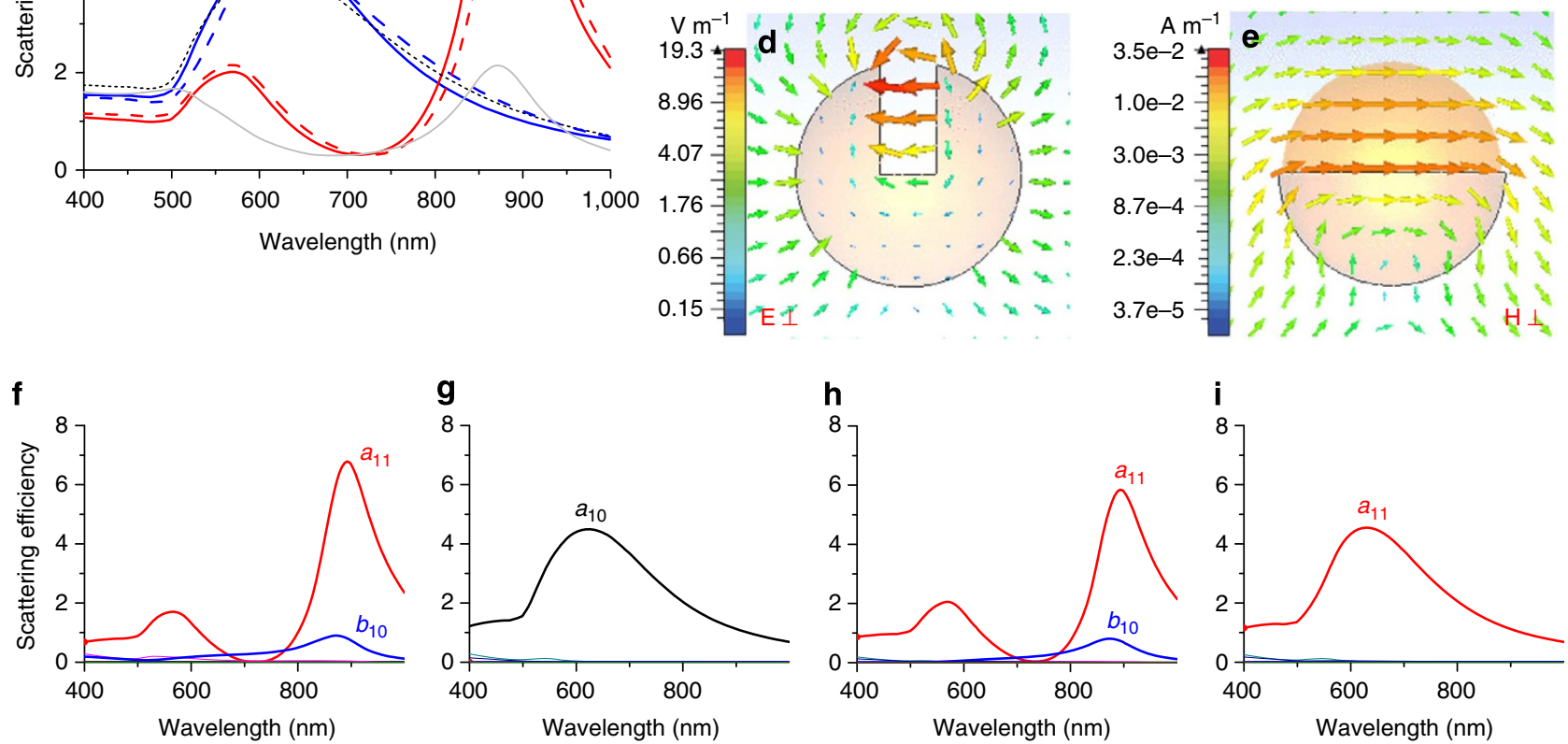

Figure 2 | Numerical simulations of optical properties of SBRs. (a) Total scattering efficiencies of an SBR formed by a $200 \mathrm{~nm}$ gold sphere with a $50 \mathrm{~nm}$ wide by $100 \mathrm{~nm}$ deep cut. The SBR is excited by a plane wave directed from top antiparallel to $z$ axis (solid curves) or from the side facing the cut along $y$ axis (dashed curves). Polarization of the incident light is perpendicular or parallel to the cut. The black dotted curve represents scattering efficiency of the same particle without a cut. The grey curve is the absorption efficiency of the SBR for top excitation with polarization perpendicular to the cut. Inset is the schematic illustration of the SBR and directions of the incident plane waves. (b-e) Distributions of electric (b,d) and magnetic $(\mathbf{c}, \mathbf{e})$ field around the SBR at electric $(\mathbf{b}, \mathbf{c})(622 \mathrm{~nm}$, polarization is parallel to the cut) and LC (d,e) (882 nm, polarization is perpendicular to the cut) resonance wavelengths. ( $\mathbf{f}-\mathbf{i})$ Decomposition of the scattered field of SBR into multipole moments based on the vector spherical harmonics. SBR is excited from the top $(\mathbf{f}, \mathbf{g})$ or from the side facing the nanocut $(\mathbf{h}, \mathbf{i})$. Incident light polarization is perpendicular $(\mathbf{f}, \mathbf{h})$ or parallel $(\mathbf{g}, \mathbf{i})$ to the cut. All the electric and magnetic spherical multipole harmonics of the first and the second order are calculated and plotted in the figure. Only harmonics that have a noticable contribution are marked by corresponding coefficients ( $b_{10}$-magnetic dipole, $a_{10}$ and $a_{11}$-electric dipoles). $a_{11}$ in all plots stays for a sum of $a_{11}$ and $a_{1-1}$ mode contributions.

is strongly enhanced and oscillates inside the nanocut parallel to its axis (Fig. 2e).

Similar spectral behaviour can also be observed in the SBR excited from the side facing the nanocut (along $y$ axis) with light polarization parallel (along $z$ axis) and perpendicular (along $x$ axis) to the cut (Fig. 2a, dashed lines). Intensities and spectral positions of both electric and magnetic LC resonances in this case are almost unchanged compared with the case of excitation from the top. This shows that the magnetic response of SBRs is almost independent of the direction of incidence when it is varied in the nanocut plane $(y z)$ and the incident light polarization is perpendicular to the gap. This property is similar to conventional SRRs, and it can be advantageous compared with multi-layer fishnets and stripes for a design of isotropic metamaterials.

To understand better the nature of resonances excited inside the SBR for both polarizations, we perform a decomposition of the scattered field of the nanostructure into multipole moments based on the vector spherical harmonics ${ }^{28,38,39}$ (see Methods for details). Results of this analysis for different incident angles and light polarizations are presented in Fig. 2f-i. Notation $b_{10}$ corresponds to the magnetic dipole mode, while $a_{10}$ and $a_{11}$ correspond to the electric dipole modes of two different types. For the incident light polarization parallel to the nanocut, only a single electric dipole resonant mode is excited while the excitation of other modes is negligibly small (Fig. 2g,i). This conclusion fully supports our previous discussion based on the electric and magnetic field distributions inside the SBR (Fig. 2b,c). We note here that the type of the electric mode changes for different polarizations from $a_{10}$ for incidence from the top (Fig. $2 \mathrm{~g}$ ) to $a_{11}$, for incidence from the side (Fig. 2i) of the SBR. For polarization perpendicular to the nanocut, the resonance around $900 \mathrm{~nm}$ exhibits an enhancement of the magnetic dipole response $b_{10}$ for both incident angles (see Fig. 2f,h). However, the electric dipole mode $a_{11}$ is also resonantly excited at this wavelength that is caused by a strong electric field enhancement inside the nanocut (see Fig. 2d). This situation differs strongly from resonant dielectric nanoparticles where a pure magnetic dipole mode can be excited ${ }^{29-35}$. However, it is similar to conventional SRRs, 
where electric field enhancement inside the gap contributes to the scattering 27,28 . The relative contribution from the magnetic dipole mode to the scattering efficiency at LC resonance with respect to the electric dipole mode in SBR is comparable with and even stronger than that for conventional SRRs ${ }^{28}$ (see Fig. 3).

In Fig. 3, we also present the analysis of similar modes for light scattering by two SRR structures with the same size of the nanogap. One of the structures is a nanocube with the side length of $200 \mathrm{~nm}$, gap width of $50 \mathrm{~nm}$ and depth of $100 \mathrm{~nm}$, which is a $3 \mathrm{D}$ cubic analogue of the SBR (Fig. 3a,b). The second is a flat SRR obtained from the nanocube by reducing its width down to $20 \mathrm{~nm}$ (Fig. 3c,d). Both of the nanostructures exhibit an LC-type resonance at longer wavelengths, but with a smaller magnetic mode contribution compared with the SBR. The ratio of the maximum scattering efficiency by the magnetic dipole mode $b_{10}$ to the maximum scattering efficiency by the electric dipole mode $a_{11}$ is: 0.081 in Fig. 3a; 0.095 in Fig. 3b; 0.066 in Fig. 3c; and 0.068 in Fig. 3d. These numbers are lower than the corresponding values for the SBR with a similar gap size: 0.13 in Fig. $2 \mathrm{f}$ and 0.14 in Fig. $2 \mathrm{~h}$. This means that the SBR supports a stronger magnetic dipole mode at LC-type resonance. It is also important to note that the relative contribution from the magnetic dipole to the LC resonance is somewhat higher in the $3 \mathrm{D}$ cube-like split-ring structure than in the flat SRR. This shows the advantage of 3D designs over flat nanostructures for generation of a strong magnetic dipole response.

To show how both electric and magnetic resonances behave with respect to the cut parameters of the SBR, different cut depths have been simulated at a fixed cut width of $20 \mathrm{~nm}$ (Fig. $4 \mathrm{a}$ ). The
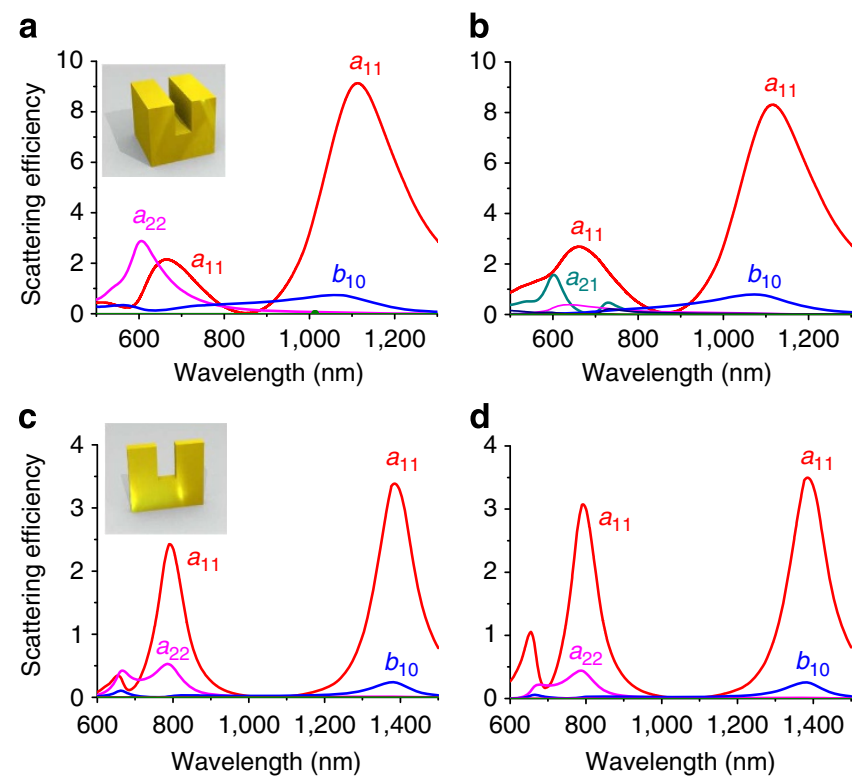

Figure 3 | Partial light scattering efficiencies by different spherical multipole modes excited inside different SRRs. $(\mathbf{a}, \mathbf{b})$ Scattering by a $3 \mathrm{D}$ gold cube with the gap on top. The cube dimentions are $200 \times 200 \times 200 \mathrm{~nm}^{3}$, the gap has the width of $50 \mathrm{~nm}$ and depth of $100 \mathrm{~nm}$. The incoming plane wave is directed from top (a) and from the side facing the cut (b). (c,d) Scattering by a flat gold SRR standing upright. The SRR has dimensions of $200 \times 200 \times 20 \mathrm{~nm}^{3}$, the gap has the width of $50 \mathrm{~nm}$ and depth of $100 \mathrm{~nm}$. The incoming plane wave is directed from top (c) and from the side facing the cut (d). All the electric and magnetic spherical multipole harmonics of the first and the second order are calculated and plotted in the figures. Only harmonics that have a noticable contribution are marked ( $a_{11}$-electric dipole, $b_{10}$-magnetic dipole, $a_{22}$ and $a_{21}$-electric quadrupoles). In case $m$ is not zero,

$a_{l m}$ in the plots stays for a sum of $a_{l m}$ and $a_{l-m}$ mode contributions. cut width has also been gradually changed at a fixed cut depth of $100 \mathrm{~nm}$ (Fig. 4b). As it is seen from the figures, both parameters strongly affects the LC resonance spectral position, while the position of the short-wavelength electric resonance is almost unchanged although its intensity is affected. When the cut depth is decreased, the LC resonance is blue-shifted, reaching the electric resonance position in the visible spectral range (Fig. 4a). This can be explained by a decrease of the capacitance of the gap (C) leading to an increase of the resonance frequency $\left(\omega^{2}=1 / L C\right)$ that is in line with the SRRs theory ${ }^{9-11}$. On the other hand, decreasing of the width of the gap increases the gap capacitance, which leads to a decrease of the resonance frequency and a corresponding red-shift of the LC resonance peak (Fig. 4b). In both these cases, the inductance $(L)$ changes slowly due to the large size of the sphere compared with the gap size. One should also note that the above LC circuit model initially developed for large SRRs is not fully applicable to our case of very small nanoparticles with nanocuts and is only used here as a simple illustration of the observed effects. Similar behaviour with respect to the depth of the gap has been observed earlier in flat split-ring resonators fabricated by focused ion beam milling of thin gold films ${ }^{12}$. It was shown that by controlling the depth of the gap, it is possible to shift the LC resonance down to the electric resonance wavelength. However, as the structures were flat, the position of both resonances was in the near-IR spectral range. In our case, the electric resonance position of the spherical particles is located
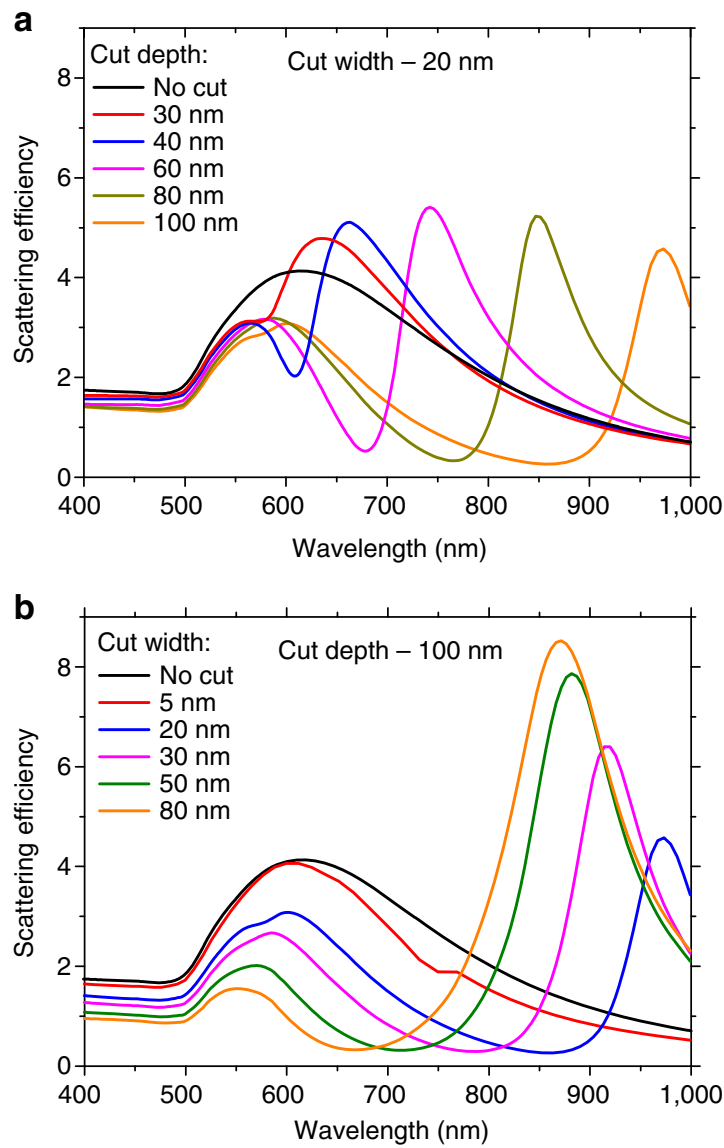

Figure 4 | Scattering properties of the SBR with varied cut parameters. The dependence of the scattering efficiency of the SBR on (a) the cut depth for a fixed cut width of $20 \mathrm{~nm}$ and (b) on the cut width at a fixed cut depth of $100 \mathrm{~nm}$. The incident plane wave is directed from top of the SBR with light polarization perpendicular to the cut. The gold sphere diameter is $200 \mathrm{~nm}$. 
in the visible spectrum that gives a possibility to shift the magnetic LC resonance down to visible wavelengths.

Experiment. To verify this concept experimentally, we first fabricated gold and silver nanoparticles using the LIT in combination with e-beam lithography ${ }^{40}$ (see Methods for more details). This method allows producing nanoparticles with almost perfect spherical shape and high surface quality. The size of the nanoparticles is controlled using an initial lithography step while their spherical shape and low surface roughness is assured by strong surface tension forces of molten metal during the laser processing. Then nanocuts with straight sidewalls have been produced on top of the nanoparticles using focused helium $(\mathrm{He})$ ion beam milling. In comparison with standard FIB systems ${ }^{41,42}$ that use gallium (Ga) ions, helium ion microscope (HIM) can provide significantly higher structuring resolution ${ }^{43-45}$. Also, in contrast to standard gallium-ion based FIB systems, HIM does not significantly dope the sidewalls of the materials during milling and thus may retain good plasmonic properties of the nanoparticles ${ }^{41,46}$

HIM images of two gold nanoparticles with cuts fabricated by this combined method are shown in Fig. 5a,b. These nanoparticles have a similar diameter of around $170 \mathrm{~nm}$ and cut width of around $15 \mathrm{~nm}$. The structured cut can be controlled in depth and width (down to $<5 \mathrm{~nm}$ ) but in this case was varied to match the modelling predictions. Variable depth was achieved by varying the dwell time at a fixed beam current. The dwell time was $25 \%$ longer for the particle shown in Fig. 5a compared with that shown in Fig. 5b, which resulted in a deeper cut (see Methods for details). Optical properties of these nanoparticles have been studied using a single-nanoparticle spectroscopy setup ${ }^{31}$. Measurements were performed in dark field geometry with illumination from one side facing the nanocut ( $k$ vector is in the
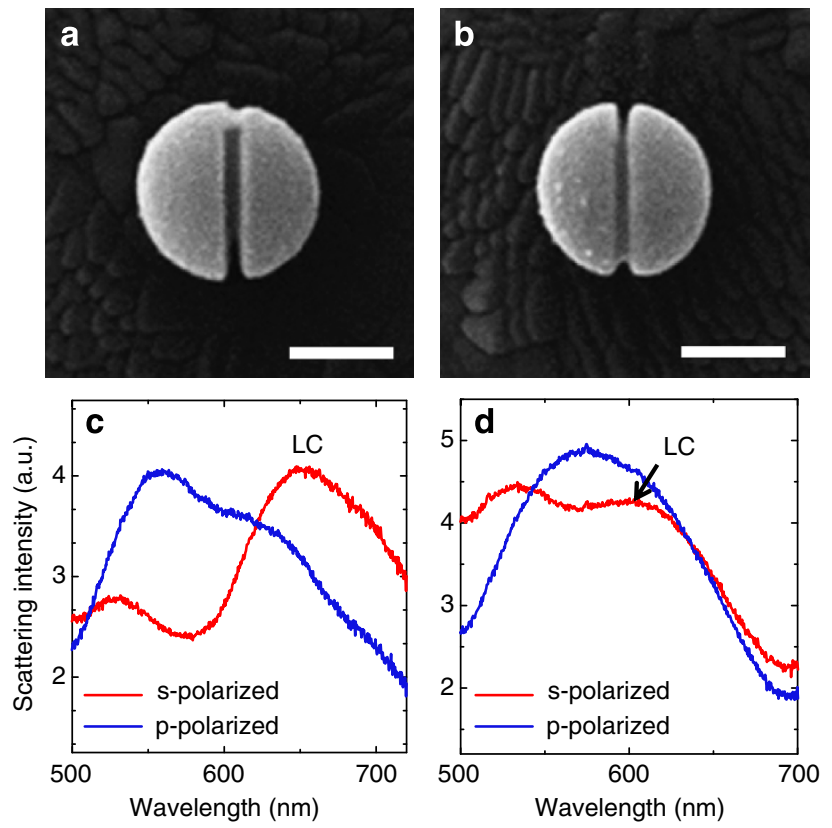

Figure 5 | Experimental realization of gold SBRs. (a,b) HIM images of two gold nanoparticles with similar diameters of $170 \mathrm{~nm}$ and cuts width of $15 \mathrm{~nm}$. Cut depth in (a) is larger than in (b), which is obtained by $25 \%$ longer helium ion beam milling time. Scale bars correspond to $100 \mathrm{~nm}$. $(\mathbf{c}, \mathbf{d})$ Scattering spectra of the particles shown in $(\mathbf{a}, \mathbf{b})$ for incident light with $\mathrm{s}$ (perpendicular to the cut) and $\mathrm{p}$ (parallel to the cut) polarizations. LC labels show spectral position of the LC resonance. It is observed at $650 \mathrm{~nm}$ in (c) and $600 \mathrm{~nm}$ in (d). $y z$ plane). Two different polarizations of excitation source have been studied: parallel and perpendicular to the nanocut (see Fig. 1 and Methods for details). In this excitation geometry, p-polarization (lying in the $y z$ plane) should only couple to the electric dipole resonance of the sphere while s-polarization (along $x$ axis) should excite both electric and magnetic dipoles. Results of these measurements for the two gold particles are shown in Fig. $5 c$,d. For p-polarization, which is parallel to the cut, only a single scattering peak corresponding to the electric dipole resonance of the sphere is observed. For s-polarization, two resonances are detected, LC resonance on the long wavelength side and a leftover of the electric dipole on the short-wavelength side. For the particle with a deeper cut (Fig. 5a,c), the LC resonance position is red-shifted compared with the particle with a shallower cut (Fig. 5b,d). These results are in a very good agreement with theoretical predictions discussed in the previous section (Fig. 4a). To the best of our knowledge, the wavelength of magnetic resonance of $600 \mathrm{~nm}$ obtained for the gold SBR in Fig. 5b,d is the shortest compared with all gold-based nanostructures reported so far.

To show that this concept may also work at even shorter wavelengths, the same approach has been applied to fabricate SBRs made of silver. Plasmonic resonance of silver nanoparticles is blue-shifted compared with gold. Thus one may expect further blue-shift of the magnetic resonance position in silver SBRs. Results for silver are shown in Fig. 6. First, a silver SBR has been fabricated with a particle diameter of around $170 \mathrm{~nm}$ and a cut width of around $15 \mathrm{~nm}$ that is similar to the gold SBRs discussed earlier (Fig. 6a). The LC resonance position in this case is around $640 \mathrm{~nm}$ (Fig. 6d) that is similar to the above results for gold nanoparticles. However, this resonance is more pronounced and well separated from the electric dipole resonance located around $500 \mathrm{~nm}$. To further shift both resonances to shorter wavelengths, a smaller silver nanoparticle with a diameter of around $80 \mathrm{~nm}$ and a $12 \mathrm{~nm}$ wide cut has been fabricated (Fig. 6b). In this case, the LC resonance position is further blue-shifted reaching the wavelength of $570 \mathrm{~nm}$, which is significantly smaller than earlier reported results obtained with SRRs ${ }^{10-13}$ and coupled nanoparticles ${ }^{18,19}$ and is comparable with the best results obtained with multi-layer stripes and fishnets ${ }^{21,22}$. However, in contrast to the stripes, fishnets and other multi-layer structures whose magnetic response can only be obtained under a single excitation direction, SBRs (similar to SRRs) possess a strong magnetic response for all incident directions lying in the plane parallel to the nanocut that may hold a bigger promise for metamaterial design and fabrication.

To show how the surface quality of the nanoparticles influences their resonant properties, HIM cut have also been introduced into a commercial chemically synthesized silver nanoparticle (NanoComposix) of $80 \mathrm{~nm}$ size (Fig. 6c,f). In this case, the electric resonance position is further blue-shifted, which may probably be explained by a higher crystallinity of the chemically synthesized silver nanoparticles. However, the LC resonance is less pronounced due to the inferior surface quality of these nanoparticles compared with the nanoparticles obtained by LIT method.

\section{Discussion}

Finally, light energy flow inside and outside the SBR at the magnetic resonance wavelength has been simulated by CST Microwave Studio software. This study is useful to understand the high scattering and absorption efficiencies of the nanostructures. Similar SBR parameters as in Fig. 2 have been used in these simulations. The Poynting vector lines at the LC resonance wavelength of $882 \mathrm{~nm}$ are shown in Fig. 7a,b in two different cross-section planes. The colour map corresponds to the 

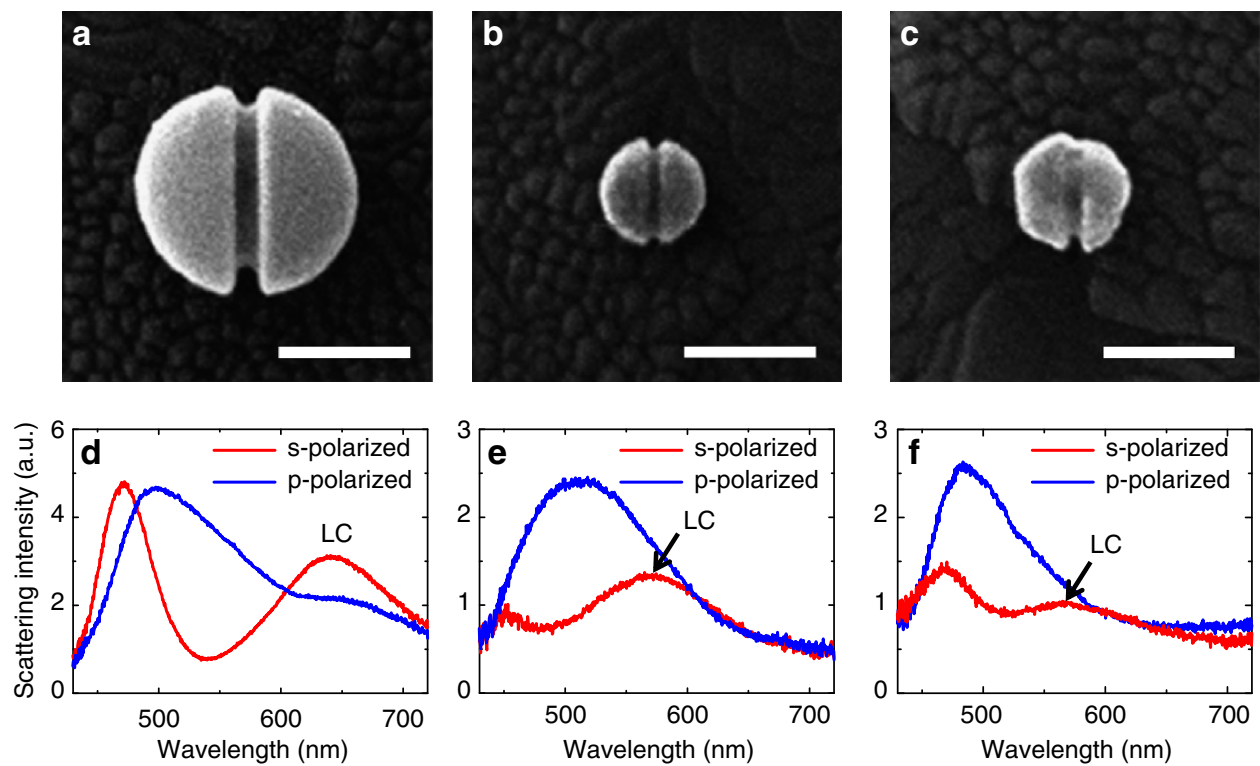

Figure 6 | Experimental realization of silver SBRs. (a-c) HIM images of three silver nanoparticles with diameters of $170 \mathrm{~nm}(\mathbf{a})$ and $80 \mathrm{~nm}(\mathbf{b}, \mathbf{c})$ and a cut width of $15 \mathrm{~nm}(\mathbf{a})$ and $12 \mathrm{~nm}(\mathbf{b}, \mathbf{c})$. The nanoparticles in $(\mathbf{a}, \mathbf{b})$ are produced by the LIT method whereas the nanoparticle in $(\mathbf{c})$ is chemically synthesized. Scale bars correspond to $100 \mathrm{~nm}$. (d-f) Scattering spectra of the nanoparticles shown in (a-c) for incident light with $\mathrm{s}$ (perpendicular to the cut) and $\mathrm{p}$ (parallel to the cut) polarizations. LC labels show spectral position of the LC resonance. It is observed at $640 \mathrm{~nm}$ in (d), $570 \mathrm{~nm}$ in (e) and $565 \mathrm{~nm}$ in (f).
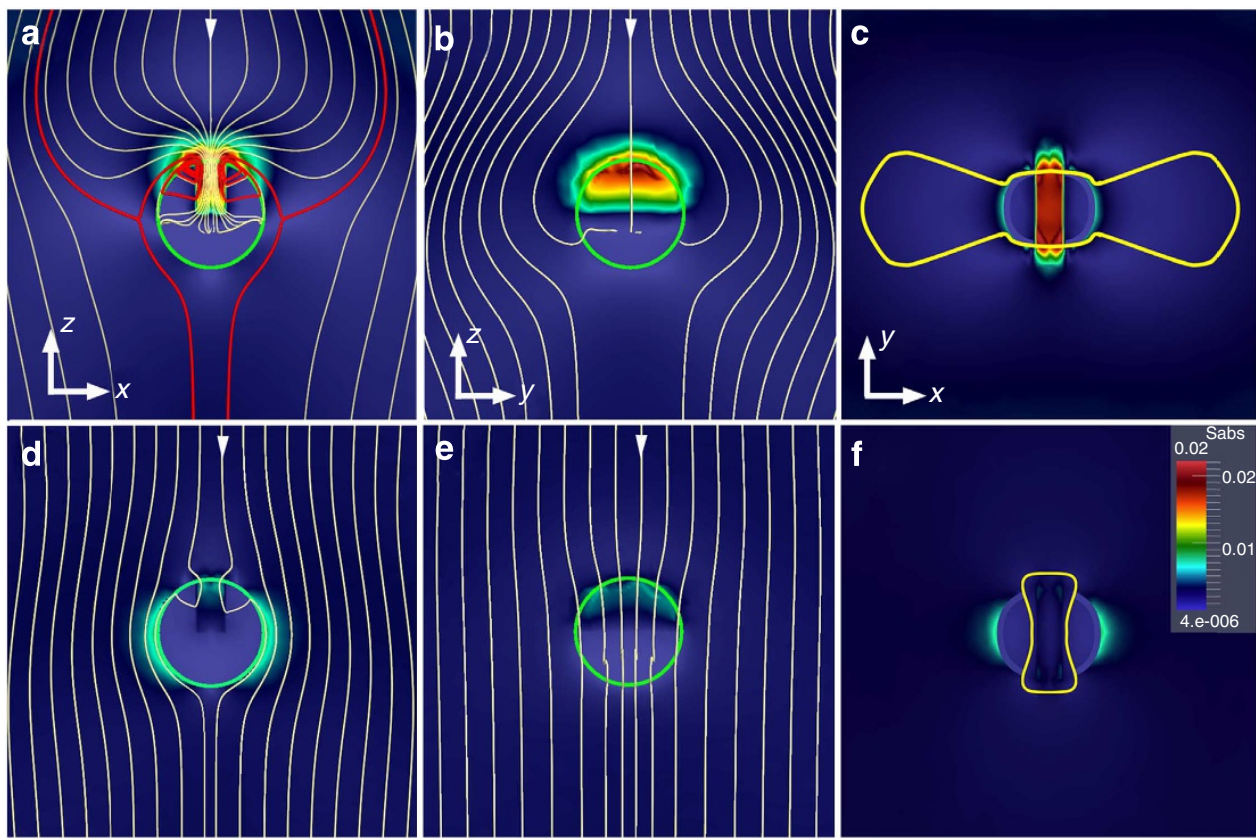

Figure 7 | Poynting vector distribution around gold SBR. (a-c) at LC resonance wavelength of $882 \mathrm{~nm}$, (d-f) at minimum scattering wavelength of $710 \mathrm{~nm}$. (a,d) show $x z$ cross-section plane in the middle of the sphere. (b,e) show $y z$ cross-section plane in the middle of the sphere. (c,f) show separatrix absorption surfaces (limited by yellow separatrix lines) of the SBR in xy cross-section plane. The SBR has $200 \mathrm{~nm}$ diameter and a cut with $50 \mathrm{~nm}$ width and $100 \mathrm{~nm}$ depth (similar to Fig. 2). Colour map represents distribution of the modulus of the Poynting vector around the nanoparticle. Red lines show separatrices of the energy flow ${ }^{47,48}$. Green circles highlight the nanoparticle location.

distribution of the modulus of the Pointing vector. It is seen in Fig. 7a that in the $x z$ plane, the Poynting vector lines spanning an area exceeding the nanoparticle cross-section by several times are collected and concentrated inside the nanocut. This generates significant field enhancement of the order of 20 inside the cut. Similar simulations performed for a silver SBR with diameter of $170 \mathrm{~nm}$ and a cut width of $15 \mathrm{~nm}$ (similar to the one shown in Fig. 6a) demonstrate even larger field enhancement inside the cut around 45 (not shown here). This energy flow passing the cut does not come out of the SBR forming optical vortices from both sides of the nanostructure. This explains the strong absorption resonance at this wavelength. On the other hand, in the $y z$ plane, most of the Poynting vector lines are strongly deviated and pass around the SBR without penetrating into it, resulting in a strong scattering efficiency at the LC resonance. Figure $2 \mathrm{c}$ shows the separatrix absorption surface of the SBR in the $x y$ plane. 
All Poynting vector lines inside the separatrix surface go into the SBR and are absorbed ${ }^{47,48}$. Strong asymmetry of the separatrix surface in $x$ and $y$ directions support our discussion above.

As a comparison, energy flow at the wavelength of the minimum scattering of $710 \mathrm{~nm}$ is also shown in the $x z$ and $y z$ planes in Fig. 7 d,e, respectively. In this case, the Poynting vector lines pass through the SBR with only a very small absorption and scattering making the nanostructure almost invisible. The separatrix surface in this case (Fig. $7 \mathrm{f}$ ) has a different geometry compared with the resonance (Fig. 7c) and is smaller than the geometrical cross-section of the particle. This behaviour is associated with destructive Fano-type interference between the electric dipole and LC resonance inside the $\mathrm{SBR}^{18,49-51}$.

In conclusion, a novel concept to realize optical magnetism at visible frequencies with standard plasmonic materials such as gold and silver is proposed. This concept is based on a spherical nanoparticle with a precision patterned nanocut. Due to a volumetric 3D shape of the proposed design a strong magnetic response tunable through the visible spectral range can be obtained. Experimentally, this unique design has been realized using laser-induced melting of nanostructures to obtain nearperfect spherical nanoparticles and helium ion beam milling to make cuts with nanometre resolution and straight sidewalls. Magnetic resonance wavelength down to $600 \mathrm{~nm}$ for gold and $565 \mathrm{~nm}$ for silver has been experimentally demonstrated here, which corresponds and exceeds previous experimental results. This novel approach to engineer resonant modes of perfect spherical nanoparticles opens new possibilities to design novel nanostructures with unique modes and high field enhancement regions that may be promising for applications to metamaterials, surface-enhanced Raman scattering, heat-assisted magnetic recording, and nanoantennas.

\section{Methods}

E-beam nanofabrication. Gold and silver nanodisks with various diameters were fabricated on quartz substrates by electron beam lithography (Elonix 100KV EBL system). First, $120 \mathrm{~nm}$ positive resist (ZEP) was coated on the sample. After baking it at $180^{\circ} \mathrm{C}$ for $2 \mathrm{~min}$, a $20 \mathrm{~nm}$ Espacer was coated on the top of the resist to avoid a charging effect. The structural geometries were defined by electron beam in the resist followed by a standard development. Then a thin Ti film $(2 \mathrm{~nm}$ thick $)$ and a $50 \mathrm{~nm}$ thick $\mathrm{Au}$ or Ag layer were deposited on the substrate by e-beam evaporation. Ti film was evaporated to ensure good adhesion between the Au/Ag layers and the substrate. A lift-off procedure was the last step to generate the nanodisks.

Laser processing. Following the first nanofabrication steps, femtosecond laser irradiation has been applied to the nanostructures. In these experiments, commercial $1 \mathrm{kHz}$ femtosecond laser system (Tsunami + Spitfire, Spectra Physics) delivering $1 \mathrm{~mJ}, 100 \mathrm{fs}$ laser pulses at a central wavelength of $800 \mathrm{~nm}$ has been used. The laser beam with a diameter of $4 \mathrm{~mm}$ was directed onto a square-shaped pinhole with $300 \mu \mathrm{m}$ size. The beam just after passing through the pinhole was imaged onto the sample surface using a $\times 20$ long distance microscope objective (Mitutoyo, MPlan NIR 20) and a $200 \mathrm{~mm}$ focus tube lens. This image transfer results in a square shape $15 \mu \mathrm{m}$ size flattop laser beam profile on the sample surface. The laser beam power was attenuated by a neutral glass filter $(15 \%)$ and a half-wave plate + polarizer to get the fluence of around $0.5 \mathrm{~J}$ per $\mathrm{cm}^{2}$ on the sample surface. The nanostructures have been melted by the laser irradiation, transformed into spherical nanodroplets by surface tension forces and transferred onto an ITOcoated glass substrate. After solidification, the molten droplets form almost perfect spherical nanoparticles. More details about the LIT and its application to nanostructures and continuous metal films for nanoparticle generation can be found in refs 40,52-54.

Helium ion beam milling. The HIM (Zeiss Orion Plus) is capable of milling extremely high-resolution structures with lateral dimensions of less than $5 \mathrm{~nm}$. When combined with a sophisticated pattern generator and custom writing strategies, near arbitrary control of the surface features is possible. In this work, the helium ion beam at a current of $0.5 \mathrm{pA}$ with a landing energy of $35 \mathrm{kV}$ in conjunction with a Nabity pattern generator was employed to further structure the surfaces of the fabricated nanoparticles. The ion fluence across the cut was varied and optimized with a multi-pass writing strategy to prevent over-milling at the edges, and eliminate residual metal redeposition while maintaining the pattern fidelity. The beam dwell time was modulated throughout the length of the cut, with commensurately longer milling time at the centre of the cut compared with the edges to compensate for the centre's increased metal thickness, as well as mitigate secondary effects such as decreased redeposition at the edges. For the gold particle in Fig. $5 \mathrm{a}$, the ion fluence was about $5 \times 10^{16}$ ions per $\mathrm{cm}^{2}$ at the edges with intermediate sections having a varying fluence of $7.5 \times 10^{17}, 3.25 \times 10^{18}$

$5.25 \times 10^{18}$ and $6.75 \times 10^{18}$ ions per $\mathrm{cm}^{2}$ towards the centre. The ion fluence was decreased by $20 \%$ for the particle in Fig. 5b. For the silver particle in Fig. $6 \mathrm{a}$, the fluence at the edges was about $8 \times 10^{17}$ ions per $\mathrm{cm}^{2}$ with intermediate sections having a varying fluence of $2.5 \times 10^{18}, 3.75 \times 10^{18}, 5 \times 10^{18}$ ions per $\mathrm{cm}^{2}$ and $8 \times 10^{18}$ ions per $\mathrm{cm}^{2}$ towards the centre. Silver particles in Fig. $6 \mathrm{~b}, \mathrm{c}$ were patterned with a fluence of $5 \times 10^{17}$ ions per $\mathrm{cm}^{2}$ at the edges, $2.5 \times 10^{18}$ ions per $\mathrm{cm}^{2}$ at the centre and a gradually increasing fluence of $1 \times 10^{18}, 1.5 \times 10^{18}$ and $2 \times 10^{18}$ ions per $\mathrm{cm}^{2}$ in between.

Single-nanoparticle spectroscopy. Spectral analysis of the fabricated SBRs was performed using a single-nanoparticle spectroscopy setup (see ref. 31 for details) in dark field geometry (Fig. 1). The sample was irradiated by a halogen lamp source from one side facing the nanocut at an angle of $58.5^{\circ}$ to the surface normal (see ref. 34 for details of dark field spectral measurements). The scattering by the nanostructure has been collected from the top into a solid angle corresponding to the microscope objective lens with $0.55 \mathrm{NA}$. Two different incident light polarizations have been studied: s-polarization-perpendicular to the nanocut and parallel to the substrate surface, and p-polarization-parallel to the nanocut and at an angle to the substrate surface (Fig. 1). The collected scattering spectra for both polarizations are normalized to the halogen lamp spectrum measured in bright-field transmission geometry. This normalization method provides correct shapes of the scattering spectra without keeping information on total scattering amplitudes.

Theoretical and numerical analysis. Numerical simulations of the SBRs were performed using commercial CST Microwave Studio software. Scattering and absorption efficiencies by the nanostructures were obtained by dividing the corresponding cross-sections by the overall geometrical cross-section of the nanoparticle.

To calculate relative contributions of different modes excited inside the SBR, we perform a decomposition of the scattered field of the nanostructure into multipole moments based on the vector spherical harmonics ${ }^{28,38,39}$. To do that, we calculated electric $a_{l-m}$ and magnetic $b_{l m}$ multipole coefficients via the electric scattered field $\mathbf{E}_{s}$ on a spherical surface enclosing the split ball centred at the origin (centre of the split ball) as follows:

$$
\begin{gathered}
a_{l m}=\frac{(-i)^{l+1} k R}{h_{l}^{(1)}(k R) E_{0} \sqrt{\pi(2 l+1) l(l+1)}} \int_{0}^{2 \pi} \int_{0}^{\pi} Y_{l m}^{*}(\theta, \phi) \hat{\mathbf{r}} \cdot \mathbf{E}_{s}(\mathbf{r}) \sin \theta \mathrm{d} \theta \mathrm{d} \phi \\
b_{l m}=\frac{(-i)^{l} k R}{h_{l}^{(1)}(k R) E_{0} \sqrt{\pi(2 l+1)}} \int_{0}^{2 \pi} \int_{0}^{\pi} \mathbf{X}_{l m}^{*}(\theta, \phi) \cdot \mathbf{E}_{s}(\mathbf{r}) \sin \theta \mathrm{d} \theta \mathrm{d} \phi
\end{gathered}
$$

where $R$ is the radius of the enclosing sphere, $k$ is the wavevector, $h_{l}{ }^{(1)}$ is the Hankel function with the asymptotics of the outgoing spherical wave, $E_{0}$ is the amplitude of the incident wave, $Y_{l m}$ and $\mathbf{X}_{l m}$ are scalar and vector spherical harmonics ${ }^{39}$. Based on these multipole coefficients, we can also calculate partial scattering efficiencies associated with a particular spherical harmonic

$$
Q_{l m}^{s c a, E}=\frac{1}{(k d)^{2}}(2 l+1)\left|a_{l m}\right|^{2}, \quad Q_{l m}^{s c a, H}=\frac{1}{(k d)^{2}}(2 l+1)\left|b_{l m}\right|^{2},
$$

where $d$ is the outer radius of the split ball. The total scattering efficiency can be obtained by taking the full sum of all partial contributions:

$$
Q^{s c a}=\sum_{l=1}^{\infty} \sum_{m=-l}^{l} Q_{l m}^{s c a, E}+Q_{l m}^{s c a, H}
$$

Although our system does not exhibit pure azimuthal symmetry, the numerical results demonstrate that mulitpole coefficients still hold the following relations: $\left|a_{l, m}\right|=\left|a_{l,-m}\right|$ and $\left|b_{l, m}\right|=\left|b_{l,-m}\right|$ that can be explained due to a relatively narrow nanocut in the split ball. Based on these results we can introduce the effective scattering efficiencies

$$
\begin{gathered}
\tilde{Q}_{l m}^{s c a, E}=Q_{l, m}^{s c a, E}+Q_{l,-m}^{s c a, E}=\frac{2}{(k d)^{2}}(2 l+1)\left|a_{l m}\right|^{2}, \\
\tilde{Q}_{l m}^{s c a, H}=Q_{l, m}^{s c a, H}+Q_{l,-m}^{s c a, H}=\frac{2}{(k d)^{2}}(2 l+1)\left|b_{l m}\right|^{2},
\end{gathered}
$$

which are the sum of two contribution for $(l, m)$ and $(l,-m)$ harmonics for $m \neq 0$. These efficiencies were plotted in Fig. 2f-i for all electric and magnetic modes of the first and the second order (dipoles and quadrupoles). Electric and magnetic dipole modes, which have the strongest contribution to the total scattering, are marked in Fig. 2 as: $b_{10}$-magnetic dipole mode, and $a_{10}$ and $a_{11}$-electric dipole modes. 


\section{References}

1. Soukoulis, C. M. \& Wegener, M. Past achievements and future challenges in the development of three-dimensional photonic metamaterials. Nat. Photon. 5, 523-530 (2011).

2. Boltasseva, A. \& Atwater, H. A. Low-loss plasmonic metamaterials. Science 331, 290-291 (2011).

3. Soukoulis, C. M. \& Wegener, M. Optical metamaterials - more bulky and less lossy. Science 330, 1633-1634 (2010).

4. Zheludev, N. I. The road ahead for metamaterials. Science 328, 582-583 (2010).

5. Shalaev, V. M. Optical negative-index metamaterials. Nat. Photon. 1, 41-47 (2007).

6. Pendry, J. B., Holden, A. J., Robbins, D. J. \& Stewart, W. J. Magnetism from conductors and enhanced nonlinear phenomena. IEEE Trans. Microwave Theory Tech. 47, 2075-2084 (1999).

7. Smith, D. R., Padilla, W. J., Vier, D. C., Nemat-Nasser, S. C. \& Schultz, S. Composite medium with simultaneously negative permeability and permittivity. Phys. Rev. Lett. 84, 4184-4187 (2000).

8. Yen, T. J. et al. Terahertz magnetic response from artificial materials. Science 303, 1494-1496 (2004).

9. Linden, S. et al. Photonic metamaterials: magnetism at optical frequencies. IEEE J. Sel. Top. Quant. Electron. 12, 1097-1105 (2006).

10. Klein, M. W., Enkrich, C., Wegener, M., Soukoulis, C. M. \& Linden, S. Single-slit split-ring resonators at optical frequencies: limits of size scaling. Opt. Lett. 31, 1259-1261 (2006).

11. Zhou, J. et al. Saturation of the magnetic response of split-ring resonators at optical frequencies. Phys. Rev. Lett. 95, 223902 (2005).

12. Enkrich, C. et al. Focused-ion-beam nanofabrication of near-infrared magnetic metamaterials. Adv. Mater. 17, 2547-2549 (2005).

13. Rockstuhl, C. et al. Resonances of split-ring resonator metamaterials in the near infrared. Appl. Phys. B 84, 219-227 (2006).

14. Dolling, G. et al. Cut-wire pairs and plate pairs as magnetic atoms for optical metamaterials. Opt. Lett. 30, 3198-3200 (2005).

15. Shalaev, V. M. et al. Negative index of refraction in optical metamaterials. Opt. Lett. 30, 3356-3358 (2005).

16. Zhang, S. et al. Experimental demonstration of near-infrared negative-index metamaterials. Phys. Rev. Lett. 95, 137404 (2005).

17. Dolling, G., Enkrich, C., Wegener, M., Soukoulis, C. M. \& Linden, S. Simultaneous negative phase and group velocity of light in a metamaterial. Science 312, 892-894 (2006).

18. Shafiei, F. et al. A subwavelength plasmonic metamolecule exhibiting magneticbased optical Fano resonance. Nat. Nanotech. 8, 95-99 (2013).

19. Sheikholeslami, S. N., Alaeian, H., Koh, A. L. \& Dionne, J. A. A metafluid exhibiting strong optical magnetism. Nano Lett. 13, 4137-4141 (2013).

20. Dolling, G., Wegener, M., Soukoulis, C. M. \& Linden, S. Negative-index metamaterial at $780 \mathrm{~nm}$ wavelength. Opt. Lett. 32, 53-55 (2007).

21. Cai, W. et al. Metamagnetics with rainbow colors. Opt. Express 15, 3333-3341 (2007).

22. Xiao, S., Chettiar, U. K., Kildishev, A. V., Drachev, V. P. \& Shalaev, V. M. Yellow-light negative-index metamaterials. Opt. Lett. 34, 3478-3480 (2009).

23. Lahiri, B., McMeekin, S. G., Khokhar, A. Z., De La Rue, R. M. \& Johnson, N. P. Magnetic response of split ring resonators (SRRs) at visible frequencies. Opt. Express 18, 3210-3218 (2010).

24. Garcia-Meca, C. et al. Low-loss multilayered metamaterial exhibiting a negative index of refraction at visible wavelengths. Phys. Rev. Lett. 106, 067402 (2011).

25. Okamoto, T., Otsuka, T., Sato, S., Fukuta, T. \& Haraguchi, M. Dependence of LC resonance wavelength on size of silver split-ring resonator fabricated by nanosphere lithography. Opt. Express 20, 24059-24067 (2012).

26. Tomioka, T., Kubo, S., Nakagawa, M., Hoga, M. \& Tanaka, T. Split-ring resonators interacting with a magnetic field at visible frequencies. Appl. Phys. Lett. 103, 071104 (2013).

27. Zhou, L. \& Chui, S. T. Eigenmodes of metallic ring systems: a rigorous approach. Phys. Rev. B 74, 035419 (2006).

28. Muehlig, S., Menzel, C., Rockstuhl, C. \& Lederer, F. Multipole analysis of metaatoms. Metamaterials 5, 64-73 (2011).

29. Evlyukhin, A. B., Reinhardt, C., Seidel, A., Luk'yanchuk, B. S. \& Chichkov, B. N. Optical response features of Si-nanoparticle arrays. Phys. Rev. B 82, 045404 (2010).

30. Garcia-Etxarri, A. et al. Strong magnetic response of submicron silicon particles in the infrared. Opt. Express 19, 4815-4826 (2011).

31. Kuznetsov, A. I., Miroshnichenko, A. E., Fu, Y. H., Zhang, J. B. \& Luk'yanchuk, B. Magnetic Light. Sci. Rep. 2, 492 (2012).

32. Evlyukhin, A. B. et al. Demonstration of magnetic dipole resonances of dielectric nanospheres in the visible region. Nano Lett. 12, 3749-3755 (2012).

33. Geffrin, J. M. et al. Magnetic and electric coherence in forward- and backscattered electromagnetic waves by a single dielectric subwavelength sphere. Nat. Commun. 3, 1171 (2012).

34. Fu, Y. H., Kuznetsov, A. I., Miroshnichenko, A. E., Yu, Y. F. \& Luk'yanchuk, B. Directional visible light scattering by silicon nanoparticles. Nat. Commun. 4, 1527 (2013).
35. Person, S. et al. Demonstration of zero optical backscattering from single nanoparticles. Nano Lett. 13, 1367-1868 (2013).

36. Chen, W. T. et al. Optical magnetic response in three-dimensional metamaterial of upright plasmonic meta-molecules. Opt. Express 19, 12837-12842 (2011).

37. Staude, I. et al. Hybrid high-resolution three-dimensional nanofabrication for metamaterials and nanoplasmonics. Adv. Mater. 25, 1260-1264 (2013).

38. Jackson, J. D. Classical Electrodynamics 3rd edn (Wiley, 1998).

39. Grahn, P., Shevchenko, A. \& Kaivola, M. Electromagnetic multipole theory for optical nanomaterials. New J. Phys. 14, 093033 (2012).

40. Kuznetsov, A. I. et al. Laser fabrication of large-scale nanoparticle arrays for sensing applications. ACS Nano. 5, 4843-4849 (2011).

41. Leen, J. B., Hansen, P., Cheng, Y.-T. \& Hesselink, L. Improved focused ion beam fabrication of near-field apertures using a silicon nitride membrane. Opt. Lett. 33, 2827-2829 (2008).

42. Gervinskas, G., Seniutinas, G., Rosa, L. \& Juodkazis, S. Arrays of arbitrarily shaped nanoparticles: overlay-errorless direct ion write. Adv. Optical Mater. 1, 456-459 (2013).

43. Ananth, M. et al. Creating nanohole arrays with the helium ion microscope. Proc. SPIE 8036, 80360M (2011).

44. Scipioni, L., Ferranti, D. C., Smentkowski, V. S. \& Potyrailo, R. A. Fabrication and initial characterization of ultrahigh aspect ratio vias in gold using the helium ion microscope. J. Vac. Sci. Tech. B 28, C6P18 (2010).

45. Scholder, O. et al. Helium focused ion beam fabricated plasmonic antennas with sub-5 nm gaps. Nanotechnology 24, 395301 (2013).

46. Pickard, D. S. A Distributed Axis Electron Beam System for High-Speed Lithography and Defect Inspection. PhD Thesis (Stanford University, 2006).

47. Luk'yanchuk, B. S., Miroshnichenko, A. E. \& Kivshar, Y. u. S. Fano resonances and topological optics: an interplay of far- and near-field interference phenomena. J. Opt. 15, 073001 (2013).

48. Rahmani, M. et al. Beyond the hybridization effects in plasmonic nanoclusters diffraction-induced enhanced absorption and scattering. Small doi:10.1002/ smll.201301419 (2013)

49. Luk'yanchuk, B. et al. The Fano resonance in plasmonic nanostructures and metamaterials. Nat. Mater. 9, 707-715 (2010).

50. Miroshnichenko, A. E., Flach, S. \& Kivshar, Y. S. Fano resonances in nanoscale structures. Rev. Mod. Phys. 82, 2257-2298 (2010).

51. Yang, J., Rahmani, M., Teng, J. H. \& Hong, M. H. Magnetic-electric interference in metal-dielectric-metal oligomers: generation of magneto-electric Fano resonance. Opt. Mater. Express 2, 1407-1415 (2012).

52. Kuznetsov, A. I., Koch, J. \& Chichkov, B. N. Laser-induced backward transfer of gold nanodroplets. Opt. Express 17, 18820-18825 (2009).

53. Kuznetsov, A. I. et al. Laser-induced transfer of metallic nanodroplets for plasmonics and metamaterial applications. J. Opt. Soc. Am. B 26, B130-B138 (2009).

54. Kuznetsov, A. I., Kiyan, R. \& Chichkov, B. N. Laser fabrication of 2D and 3D microstructures from metal nanoparticles. Opt. Express 18, 21198-21203 (2010).

\section{Acknowledgements}

Yiguo Chen (DSI) is acknowledged for help with laser nanoparticle fabrication at the initial stage of this work. Kwaku Eason (DSI) is acknowledged for editing. This work was supported by the Singapore National Research Foundation project NRF2011NRFCRP002-050, by the National University of Singapore ODPRT funded project R263000674133, by the Agency for Science, Technology and Research (A*STAR) of Singapore: SERC Grant 1021520011 and by the Australian Research Council through the AEM Future Fellowship project FT110100037.

\section{Author contributions}

A.I.K. contributed to initial idea generation, laser processing, CST simulations and spectral characterization; he coordinated the whole work and wrote the paper. A.E.M. contributed to initial idea generation, performed CST simulations and multipole decomposition, and contributed to the manuscript preparation. Y.H.F. performed spectral characterization. V.Vi. performed HIM milling and imaging. M.R. performed e-beam nanofabrication. V.Va. and Z.Y.P. performed the laser processing. Y.K. supervised numerical simulations. D.S.P. supervised HIM work. B.L. supervised the whole work. All authors read and corrected the manuscript before the submission.

\section{Additional information}

Competing financial interests: The authors declare no competing financial interests.

Reprints and permission information is available online at http://npg.nature.com/ reprintsandpermissions/

How to cite this article: Kuznetsov, A. I. et al. Split-ball resonator as a three-dimensional analogue of planar split-rings. Nat. Commun. 5:3104 doi: 10.1038/ncomms4104 (2014). 